\title{
Strongly coupled single quantum dot in a photonic crystal waveguide cavity
}

\author{
F. S. F. Brossard, ${ }^{1, a)}$ X. L. $X u{ }^{1}{ }^{\text {D. A. Williams, }},{ }^{1}$ M. Hadjipanayi, ${ }^{2}$ M. Hugues, ${ }^{3}$ \\ M. Hopkinson, ${ }^{3}$ X. Wang, ${ }^{2}$ and R. A. Taylor ${ }^{2}$ \\ ${ }^{1}$ Hitachi Cambridge Laboratory, Hitachi Europe Ltd., J. J. Thomson Avenue, Cambridge CB3 OHE, \\ United Kingdom \\ ${ }^{2}$ Department of Physics, University of Oxford, Parks Road, Oxford OX1 3PU, United Kingdom \\ ${ }^{3}$ Department of Electronic and Electrical Engineering, University of Sheffield, Mapping Street. Sheffield S1 \\ 3JD, United Kingdom
}

(Received 15 March 2010; accepted 18 August 2010; published online 13 September 2010)

\begin{abstract}
Cavities embedded in photonic crystal waveguides offer a promising route toward large scale integration of coupled resonators for quantum electrodynamics applications. In this letter, we demonstrate a strongly coupled system formed by a single quantum dot and such a photonic crystal cavity. The resonance originating from the cavity is clearly identified from the photoluminescence mapping of the out-of-plane scattered signal along the photonic crystal waveguide. The quantum dot exciton is tuned toward the cavity mode by temperature control. A vacuum Rabi splitting of $\sim 140 \mu \mathrm{eV}$ is observed at resonance. (C) 2010 American Institute of Physics.
\end{abstract}

[doi:10.1063/1.3487937]

Photonic crystal waveguide $(\mathrm{PhCWG})$ cavities provide the highest quality factors $(Q$ 's) reported to date among microcavities with a mode volume $V$ of the order of $\left(\lambda_{0} / n\right)^{3}$.,2 The resulting very large $Q / V$ makes them particularly suitable candidates for studying strong light-matter interactions. In addition, the PhCWG section can be tailored to form large-scale arrays of low-loss coupled resonators with the possibility of in-line input-output coupling as recently demonstrated. ${ }^{3}$ The strong coupling regime with a single quantum dot (QD) has been demonstrated in $\mathrm{H} 1$ or L3 type cavities which are isolated from the output-interface of the $\mathrm{PhC}$ by the photonic lattice. ${ }^{4,5}$ The demonstration of the strong coupling regime in a cavity embedded in a PhCWG would constitute an important step toward the realization of quantum computing devices based on an array of coupled photonic microcavities. ${ }^{6,7}$

The PhCWG cavity studied here is based on the local modulation of the PhCWG width. ${ }^{2}$ It was designed to emit resonantly at the low energy tail of a high dot density sample such as to limit absorption effects due to the QD ensemble and light losses related to fabrication imperfections. The holes were shifted slightly and gradually away from the $\mathrm{Ph}$ CWG as indicated in Fig. 1(a) by a distance adapted from Ref. 2. The gradual shift of the holes by a few nm creates a low-loss resonant mode confined by the mode gap of the PhCWG, based on the same principle as that used in a double heterostructure. ${ }^{1}$ The device was fabricated by first defining the $\mathrm{PhC}$ pattern lithographically in poly(methyl methacrylate) with a $100 \mathrm{kV}$ VB6 Leica e-beam machine. The pattern was then transferred onto a 180-nm-thick GaAs slab containing InAs QDs using reactive ion etching (RIE) with a $\mathrm{SiCl}_{4} / \mathrm{Ar}$ mixture; the remaining resist was removed with a $\mathrm{NH}_{4} \mathrm{OH} /$ acetone soak before $\mathrm{HF}$ treatment forming an air-bridge. Finally, a digital etch step was employed using $\mathrm{O}_{2} / \mathrm{HCl}$ to remove any damaged native oxide resulting from the RIE procedure. ${ }^{8}$ A scanning electron microscope (SEM)

${ }^{a)}$ Electronic mail: fsfb2@cam.ac.uk. image of the fabricated cavity is shown in Fig. 1(a) and a view of the entire PhCWG structure with no apparent disorder shown in Fig. 1(b). The expected cavity mode profile, $Q$, and mode volume were obtained using a freely available three-dimensional finite difference time domain (FDTD) package MEEP. ${ }^{9}$ A $Q$ of about $2 \times 10^{7}$ and a $V \sim 1.3\left(\lambda_{0} / n\right)^{3}$ for $n=3.46$ (Ref. 10) was found using flux planes surrounding the PhCWG with the same size as the fabricated device. The electric field energy distribution shown at the center of the cavity in Fig. 1(c) confirms that such a design should support an ultrahigh $Q$ cavity mode.

Photoluminescence (PL) measurements were carried out on a high InAs dot density $\left(\sim 100 \operatorname{dots} / \mu \mathrm{m}^{2}\right)$ wafer with
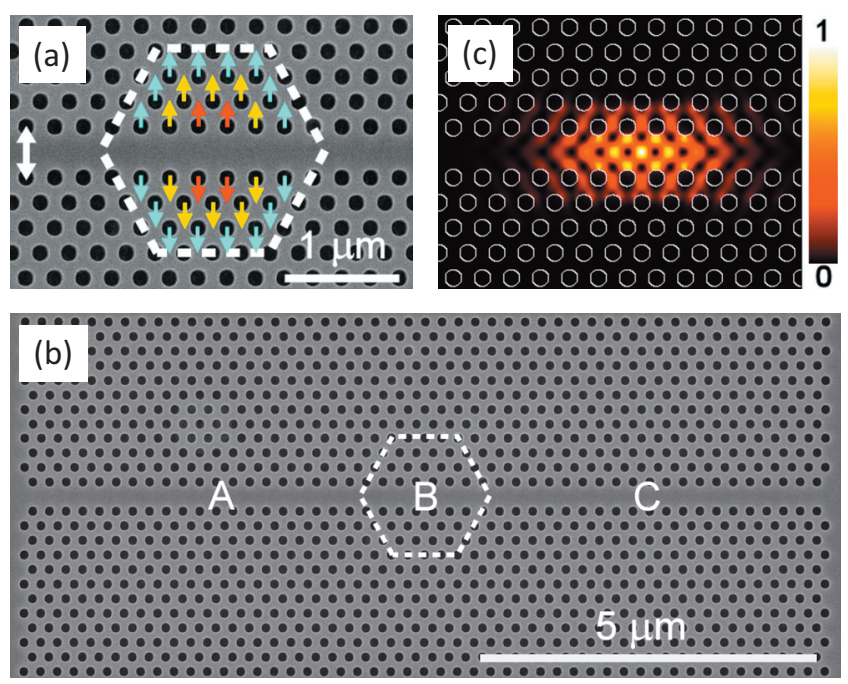

FIG. 1. (Color) (a) SEM image of the cavity embedded in a PhCWG with width $W=0.98 \sqrt{3} a$ indicated by the white double arrow, where $a$ is the lattice constant. The holes are shifted within the dashed hexagon by (red arrows) $6 \mathrm{~nm}$, (yellow arrows) $4 \mathrm{~nm}$, and (blue arrows) $2 \mathrm{~nm}$ in a $250 \mathrm{~nm}$ $\mathrm{PhC}$ lattice with hole size of about $140 \mathrm{~nm}$. (b) zoomed out SEM image of (a) showing the symmetrically defined left (a) and right (c) PhCWG sections and the cavity defined at B. (c) Calculated electric field energy distribution of the cavity mode. 

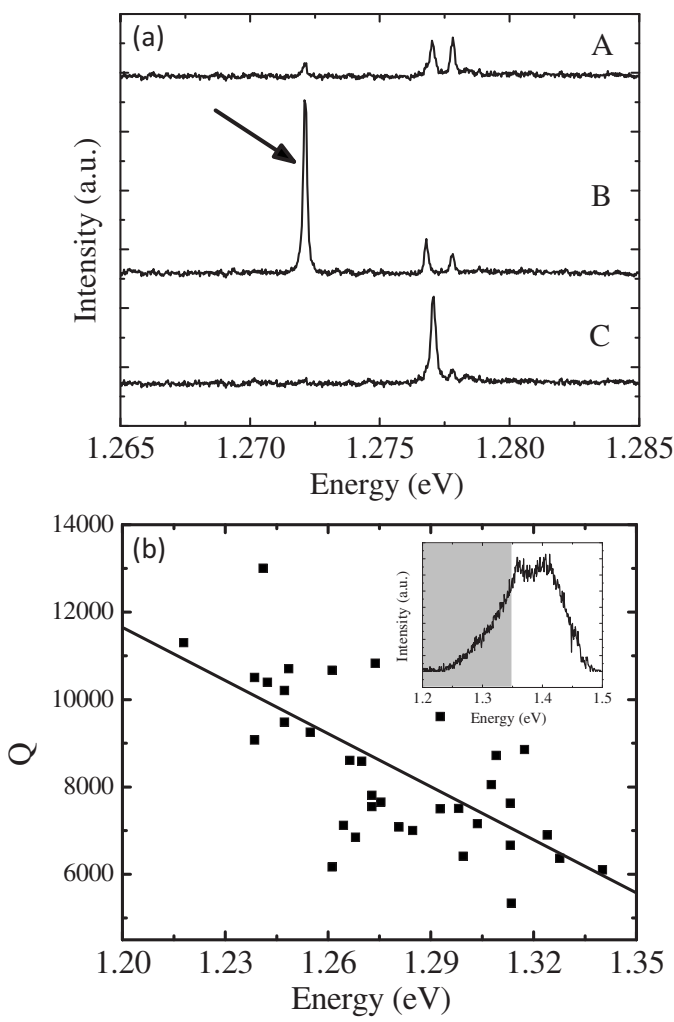

FIG. 2. (a) PL spectra of the resonances as the laser spot is shifted through the cavity with a $50 \mathrm{~nm}$ resolution XYZ stage attached to the objective. A, $\mathrm{B}$, and $\mathrm{C}$ correspond to the sections of the PhCWG indicated in Fig. 1(b). The cavity resonance indicated by the arrow clearly disappears either sides of the cavity. (b) Recorded $Q$ of the cavity resonance for various $a$ and $r / a$. The guide-to-the-eye solid line shows the pronounced increased cavity losses with higher energies. The inset shows the PL of the QD ensemble. The range of energies over which the cavities were studied is shown by the gray area in the inset.

dots grown by molecular beam epitaxy giving a broad emission centered at $1.35 \mathrm{eV}$. The sample was mounted in a Heflow cryostat cooled to $5 \mathrm{~K}$ and the dots pumped with a $\sim 1 \mu \mathrm{m}$ spot size $\mathrm{He}-\mathrm{Ne}$ laser obtained using a $100 \times$ microscope objective (numerical aperture 0.75 ). The emission light was collected by the same objective, dispersed through a $0.55 \mathrm{~m}$ spectrometer and detected with a cooled chargecoupled device camera. A typical PL spectrum obtained with this cavity is shown in Fig. 2(a) under a pumping power of $32 \mu \mathrm{W}$. The spectrum consisted of an isolated sharp peak and a series of closely spaced peaks at slightly higher energies. These features were reproduced in all the PhCWGs investigated, with peak energies determined by the $\mathrm{PhC}$ lattice constant, hence indicating resonances associated with the band structure of the device. The cavity resonance shown by the arrow in Fig. 2(a) was clearly identified by shifting the laser spot laterally along the PhCWG and through the cavity. We attribute the sharp peaks observed either side of the cavity to waveguide resonances whose origin is not clearly understood at this stage and is the subject of further study.

Cavities with various hole sizes $r$, lattice constant $a$, and ratio $r / a$ were investigated. A snapshot of our results is reported in Fig. 2(b) showing a clear trend toward lower $Q$ for devices emitting at higher energies. The $Q$ 's reported here are orders of magnitude lower than the ultrahigh values shown elsewhere in $\mathrm{Si}$ and GaAs samples for this type of cavity. ${ }^{2,11}$ However, we would like to point out that these
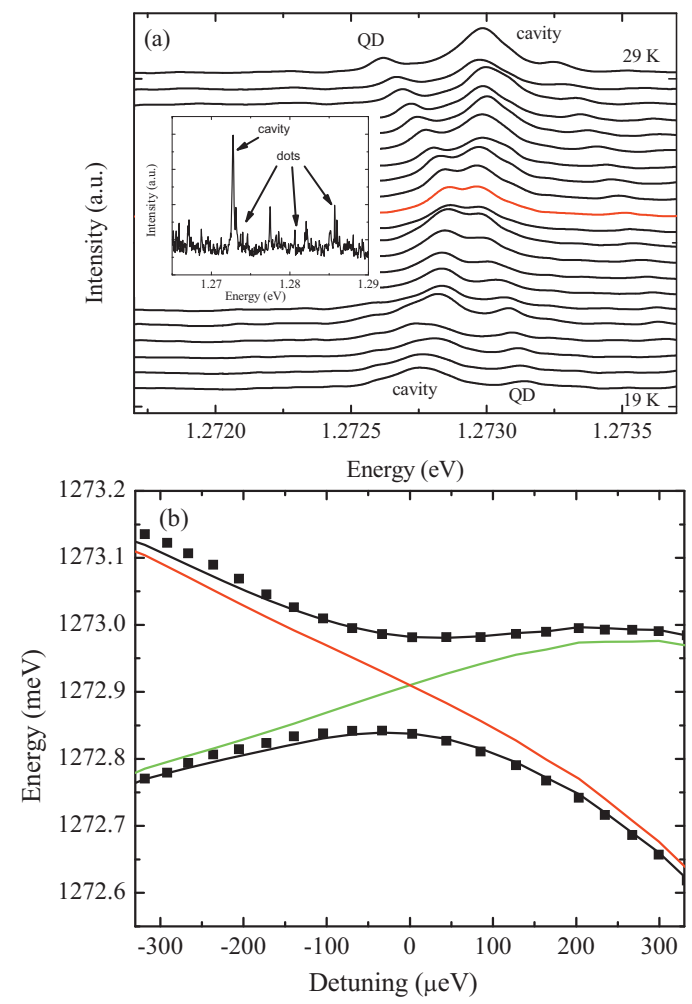

FIG. 3. (Color) (a) PL spectra of the strongly coupled QD-cavity mode system for various temperatures in steps of $0.5 \mathrm{~K}$ showing the anticrossing as the dot is tuned toward the cavity mode. The inset shows the PL spectrum over an extended energy range at $19 \mathrm{~K}$ where the cavity mode and several dots can be clearly seen. Two distinct peaks of similar line widths at resonance can be seen (red spectra). (b) Peak positions of the strongly coupled and uncoupled system for various detunings, showing a Rabi splitting of about $140 \mu \mathrm{eV}$ at zero detuning. The squares indicate the measured peaks from the strongly coupled system in (a) while the red and green lines are obtained from an uncoupled dot and cavity measured on the same sample, respectively. The black lines show the calculated peak positions for a strongly coupled system for a particular detuning energy.

ultrahigh $Q$ 's have only been reported so far for cavities emitting at lower energies than in the present work and without a layer of QDs. We believe some of the losses in our samples are associated with the more significant bulk GaAs material absorption (electron or hole traps) observed with higher energies ${ }^{12}$ as well as absorption by the QD ensemble ${ }^{13}$ whose PL is shown in the inset of Fig. 2(b).

The strong coupling regime was observed with a reduced pump power of 3.2 $\mu \mathrm{W}$ and a QD slightly to the blue of the cavity mode. The QD is strongly redshifted toward the cavity as the temperature increases due to band gap shrinkage as observed in Fig. 3(a). In an uncoupled system, both the QD and cavity photon would merge into a single peak without perturbation in their respective quantum states. In contrast, a strongly coupled system is characterized by mixed photonatom states, where the degeneracy in the energy levels of each individual entity is lifted, resulting in the Rabi splitting at resonance. This manifests itself experimentally as two distinct Lorentzian peaks and an anticrossing behavior when the dot is tuned toward the cavity, as clearly observed in our results shown in Fig. 3(a). We note that the cavity peak should redshift slightly as the temperature increases due to the increased bulk refractive index of GaAs. The blueshift observed here can be understood by considering removal of material on the surface of the sample during the heating process. We believe this material to consist of condensed gas on 
the cold sample surface. ${ }^{14} \mathrm{~A}$ Rabi splitting energy $\Delta E$ of about $140 \mu \mathrm{eV}$ is deduced from the peak positions of the dot and PhCWG cavity mode at resonance (zero detuning) as shown in Fig. 3(b). Rabi splitting occurs at resonance when the exciton-photon coupling strength becomes greater than the mean of their decay rates so that the energy can be exchanged reversibly between the QD and the cavity mode as expressed by the following: ${ }^{15}$

$$
\Delta E=2 \hbar \sqrt{g^{2}-\left(\frac{\gamma_{c}-\gamma_{x}}{4}\right)^{2}},
$$

where $g$ is the exciton-photon coupling constant, $\gamma_{c}$ and $\gamma_{x}$ are the cavity and exciton line widths, respectively. We measure $\hbar \gamma_{c}=160 \mu \mathrm{eV}$ from the full-width at half-maximum of our cavity peak at $5 \mathrm{~K}$, and $\hbar \gamma_{x}=78 \mu \mathrm{eV}$ for the single QD, the latter linewidth corresponds to the resolution limit of our spectrometer. A coupling constant $g$ of $82 \mu \mathrm{eV}$ was deduced from the fitted dot and cavity energy of the strongly coupled system shown in Fig. 3(b). We usually have $\gamma_{c} \gg \gamma_{x}$, so the condition to satisfy the strong coupling from Eq. (1) simplifies to $g>\gamma_{c} / 4$, which is clearly the case in our results. The predicted Rabi splitting can be obtained from Eq. (1) by injecting the mode volume $V$ given by the FDTD calculation and an oscillator strength $f=10.7$ for InAs QDs (Ref. 15) into the expression for the coupling strength as follows: ${ }^{15}$

$$
g=\sqrt{\frac{1}{4 \pi \varepsilon_{0} \varepsilon_{r}} \frac{\pi e^{2} f}{m V}},
$$

where $m$ is the free electron mass, $e$ the electron charge, and $\varepsilon_{0} \varepsilon_{r}$ the dielectric constants. The results show that the splitting is expected to appear in the PhCWG cavity for relatively modest $Q>3000$ and to saturate already for $Q \sim 10^{4}$, as similarly found for the L3 type cavity. ${ }^{16}$ The Rabi splitting measured in this work is about $70 \%$ of the $200 \mu \mathrm{eV}$ predicted for $Q=8000$. It is clear from previous reported results that the main obstacle in achieving the maximum splitting is the misalignment between the QD and the antinode(s) of the cavity mode, which causes the coupling strength to decrease. ${ }^{17} \mathrm{PhC}$ cavities with smaller $V$ than the PhCWG cavity have the potential to achieve larger Rabi splitting but at the expense of more stringent alignment requirements. The present system would benefit greatly from deterministic procedures to locate the position and energy of the QD for the study of coupled high $Q \mathrm{PhC}$ resonators for quantum electrodynamics applications. ${ }^{18,19}$

In conclusion, we have demonstrated the observation of the strong coupling regime between a single $\mathrm{QD}$ and a $\mathrm{Ph}$ CWG cavity formed by locally tuning the PhCWG lattice. The Rabi splitting reported compares well with previously reported results for $\mathrm{L} 3$ and $\mathrm{H} 1$ type $\mathrm{PhC}$ cavities with smaller mode volume. Future work will investigate whether the state of the exciton-photon coupled system can be probed through the resonances observed in the PhCWG. We believe the results presented here to be an important step toward multiple coupled cavities which can be addressed through the PhCWG for future quantum networks.

The FDTD computation was performed using the Darwin Supercomputer of the University of Cambridge High Performance Computing Service (http:// www.hpc.cam.ac.uk/), provided by Dell Inc. using Strategic Research Infrastructure Funding from the Higher Education Funding Council for England. This research is part of the QIP IRC supported by EPSRC (Grant No. GR/S82176/01). F. S. F. Brossard and X. L. Xu contributed equally to this work.

${ }^{1}$ B. S. Song, S. Noda, T. Asano, and Y. Akahane, Nature Mater. 4, 207 (2005).

${ }^{2}$ E. Kuramochi, M. Notomi, S. Mitsugi, A. Shinya, T. Tanabe, and T. Watanabe, Appl. Phys. Lett. 88, 041112 (2006).

${ }^{3}$ M. Notomi, E. Kuramochi, and T. Tanabe, Nat. Photonics 2, 741 (2008).

${ }^{4}$ T. Yoshie, A. Scherer, J. Hendrickson, G. Khitrova, H. M. Gibbs, G. Rupper, C. Ell, O. B. Shchekin, and D. G. Deppe, Nature (London) 432, 200 (2004).

${ }^{5}$ Y. Ota, M. Shirane, M. Nomura, N. Kumagai, S. Ishida, S. Iwamoto, S. Yorozu, and Y. Arakawa, Appl. Phys. Lett. 94, 033102 (2009).

${ }^{6}$ M. J. Hartmann, F. G. S. L. Brandão, and M. B. Plenio, Nat. Phys. 2, 849 (2006).

${ }^{7}$ D. G. Angelakis, M. F. Santos, and S. Bose, Phys. Rev. A 76, 031805(R) (2007).

${ }^{8}$ K. Hennessy, A. Badolato, A. Tamboli, P. M. Petroff, E. Hu, M. Atatüre, J. Dreiser, and A. Imamoğlu, Appl. Phys. Lett. 87, 021108 (2005).

${ }^{9}$ A. Farjadpour, D. Roundy, A. Rodriguez, M. Ibanescu, P. Bermel, J. D. Joannopoulos, S. G. Johnson, and G. Burr, Opt. Lett. 31, 2972 (2006).

${ }^{10}$ D. C. Reynolds, K. K. Bajaj, C. W. Litton, G. Peters, P. W. Yu, and J. D. Parsons, J. Appl. Phys. 61, 342 (1987).

${ }^{11}$ S. Combrié, A. De Rossi, Q. V. Tran, and H. Benisty, Opt. Lett. 33, 1908 (2008).

${ }^{12}$ C. P. Michael, K. Srinivasan, T. J. Johnson, O. Painter, K. H. Lee, K. Hennessy, H. Kim, and E. Hu, Appl. Phys. Lett. 90, 051108 (2007).

${ }^{13}$ J. Hendrickson, B. C. Richards, J. Sweet, S. Mosor, C. Christenson, D. Lam, G. Khitrova, H. M. Gibbs, T. Yoshie, A. Scherer, O. B. Shchekin, and D. G. Deppe, Phys. Rev. B 72, 193303 (2005).

${ }^{14}$ S. Mosor, J. Hendrickson, B. C. Richards, J. Sweet, G. Khitrova, H. M. Gibbs, T. Yoshie, A. Scherer, O. B. Shchekin, and D. G. Deppe, Appl. Phys. Lett. 87, 141105 (2005).

${ }^{15}$ L. C. Andreani, G. Panzarini, and J. M. Gérard, Phys. Rev. B 60, 13276 (1999).

${ }^{16}$ L. C. Andreani, D. Gerace, and M. Agio, Phys. Status Solidi B 242, 2197 (2005).

${ }^{17}$ K. Hennessy, A. Badolato, M. Winger, D. Gerace, M. Atatüre, S. Gulde, S. Fält, E. L. Hu, and A. Imamoğlu, Nature (London) 445, 896 (2007).

${ }^{18}$ K. H. Lee, A. M. Green, R. A. Taylor, D. N. Sharp, J. Scrimgeour, O. M Roche, J. H. Na, A. F. Jarjour, A. J. Turberfield, F. S. F. Brossard, D. A Williams, and G. A. D. Briggs, Appl. Phys. Lett. 88, 193106 (2006).

${ }^{19}$ A. Badolato, K. Hennessy, M. Atatüre, J. Dreiser, E. Hu, P. M. Petroff, and A. Imamoğlu, Science 308, 1158 (2005). 